Annales Geophysicae (2003) 21: 2259-2269 (C) European Geosciences Union 2003

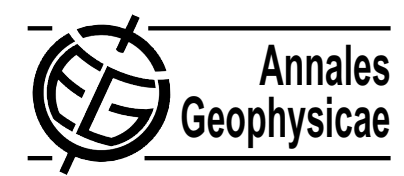

\title{
Two-dimensional current-carrying plasma sheet in the near-Earth geomagnetic tail region: a quasi-stationary evolution
}

\author{
A. V. Manankova \\ Institute of Physics, St. Petersburg University, St. Petersburg 198504, Russia \\ Received: 3 February 2003 - Revised: 11 April 2003 - Accepted: 13 May 2003
}

\begin{abstract}
A problem concerning stationary configurations of an inhomogeneous, current-carrying, two-dimensional plasma sheet as the solution of the Grad-Shafranov equation with boundary conditions given on cross-sheet profiles at the foot of the sheet and at infinity is considered, with the aim of using its solution for the description of the interaction of two current systems: the current system of the geomagnetic field, and the tail currents. The obtained solution is an exact analytical solution which contains 5 independent parameters characterizing the intensity of the current sheet. As the solution is exact, it may be applied to describe the most interesting transitional magnetospheric region: that of a strong interaction between the magnetic fields of the geodipole and of the current sheet, i.e. the region where characteristic scales of the change of all variables along and across the sheet are of the same order. This makes it possible to model the structure of the transitional region and its dynamics under quasistationary variation of the input parameters. The obtained solution describes the principal processes developing at various phases of magnetospheric disturbances, such as (1) formation of a very intense thin current sheet localized within the transition region, (2) changing from the quasi-dipolar magnetic field to the configuration when a "neck" is formed in this region. An important feature of the obtained solution is the existence of a critical value of one of the parameters of the problem, which leads to the change in the geomagnetic field configuration described above. The solution can be used as an initial condition in simulating dynamical processes in the magnetotail current sheet, as well as in testing the current sheet stability. In the summary a series of limitations in the model problem under consideration is discussed.
\end{abstract}

Key words. Magnetospheric physics (magnetotail; plasma sheet; magnetospheric configuration and dynamics)

Correspondence to: A. V. Manankova

(marianna@geo.phys.spbu.ru)

\section{Introduction}

The major purpose of the present study is to develop an exact two-dimensional mathematical description of the stationary current sheet configurations and their quasi-stationary evolution under varying geomagnetic conditions. The problem is reduced to a solution of the Grad-Shafranov equation. The region of transition from dipolar to stretched magnetic field lines is considered, which the topology determines the choice of the boundary conditions for the problem.

In general, under a two-dimensional geometry, a nonuniformity along the tail current sheet can be set in two manners: (a) via nonuniform boundary conditions along the tail (further along the $x$-axes, which is sunward directed), or (b) through different $z$-dependent boundary conditions (further along the $z$-axes, which is directed upward, perpendicular to the current sheet) on the left (near-Earth) side of the sheet and far down the tail $(x \rightarrow-\infty)$. The boundary conditions of the first type are related to the variation of the magnetopause and lobe parameters down the tail. The second type of boundary conditions reflects the relation between the internal and external magnetic field sources in the Earth's magnetosphere. Only the boundary conditions of the second type will be treated in the present study.

In the papers (Manankova et al., 2000a, b) the solutions of the Grad-Shafranov equation were examined for the boundary conditions set, as in Kan (1973): on the left boundary $(x=0)$ at the sheet centre $(z=0)$, the plasma density $n(0,0)$ and magnetic field $z$-component $B_{z}(0,0)$ are given. The solutions being sought after have to reproduce the observed distributions $B_{z}(0, x)$ and $n(0, x)$ down the tail. The choice of the near-Earth boundary values of $n(0,0)$ and $B_{z}(0,0)$ is based on the observations. Two topologically different solutions can be obtained in this case. One of them has a monotonous character and transfers to the Harris (1962) solution. The other (Manankova et al., 2000a, b) describes a spatially oscillating, inhomogeneous solution along the tail distribution of plasma and magnetic field (see also Komarov, 1963; Fadeev et al., 1965). It is clear that neither 
monotonously declining down the tail nor oscillating solution can be applied to describe the formation in the near tail of such structures as thin, intense current sheets, formation of a "neck" (a region with minimum current density and negative $B_{z}$ values), steep gradients of plasma and magnetic field distributions, etc. This makes an attempt reasonable to build a more general class of solutions of the GradShafranov equation which could be used to reproduce the structure of the near-Earth geomagnetic tail region in the quiet and disturbed conditions (Manankova and Pudovkin, 2002). A quasi-stationary evolution is modeled only as a series of stationary solutions at any fixed value of time.

\section{Formulation of the problem}

The physical model, in the frame of which there is discussed a two-dimensional, perfectly conducting, current-carrying plasma sheet (further for brevity referred to as "a plasma sheet"), is a collisionless, neutral, two-stream plasma with macroscopic velocities of charged particles $V_{i} e_{y}=-V_{e} e_{y}$, with $e_{y}$ being the unit vector along the $y$-axes, which is directed toward dusk. The velocities $V_{i}, V_{e}$ are adopted to be independent of the coordinates. It is known that such a defined plasma sheet can be treated both in kinetics (the Vlasov-Maxwell equations) and in magneto-hydrodynamics (MHD) (for details, see Manankova and Pudovkin, 1999). The problem is reduced to a solution of the Grad-Shafranov equation, which has the form

$\nabla^{2} a A(x, z)=-2 K \exp \{a A(x, z)\}$,

where $A(x, z)$ is the magnetic vector potential $y$-component, $K=4 \pi q \beta a n_{0} / m^{2}, q$ is the charge of an ion, $a=q \beta / \Theta$, $\beta=V / c, c$ is the light velocity, $V=V_{i}, \Theta=k T$ is plasma temperature in energy units; $x=m x^{*}, z=m z^{*}$ are the dimensionless coordinates, $x^{*}, z^{*}$ are dimensional coordinates, $m^{-1}$ is the characteristic length scale, which is chosen in such a way as to have $K=1$. In this case one can relate the plasma density (the quasi-neutrality is assumed, i.e. $\left(n_{i}(x, z)=n_{e}(x, z)=n(x, z)\right)$ to the vector potential $A(x, z)$ as

$n(x, z)=n_{0} \exp a A(x, z)$,

where $n_{0}$ is the constant of integration equal to the plasma density along the magnetic field line with $A(x, z(x))=0$. The choice of the constants involved in the formulation of the problem was discussed in detail by Manankova and Pudovkin (1996) in calculating the energetic parameters of the plasma sheet. Everywhere else we consider $\Theta$ to be constant.

It has to be especially noted that Eq. (1), with an exponential right-hand side, is obtained from the system of onefluid magneto-hydrodynamics (in the isothermal process approximation), taking into account that for the description of a current-carrying plasma sheet, one has to use in the system of the MHD equation Ohm's law in a generalized form. This uniquely determines the right-hand side of Eq. (1) as an exponent. This question is considered in detail in the above cited paper by Manankova and Pudovkin (1999).

The solution of Eq. (1) can be written as follows (Walcker, 1915)

$a A(x, z)=\ln \frac{4\left|f^{\prime}(\eta)\right|^{2}}{\left[1+K|f(\eta)|^{2}\right]^{2}}$,

where $f(\eta)$ is an arbitrary analytical function of the complex variable $\eta=z+i x$, with $i$ being the imaginary unity, $f^{\prime}(\eta)$ is the derivative of this function with respect to $\eta$. In the case of $f(\eta)$ being analytical, we can write: $f(\eta)=\tilde{u}(x, z)+$ $i \tilde{v}(x, z)$, where $\tilde{u}(x, z)=\operatorname{Ref}(\eta), \tilde{v}(x, z)=\operatorname{Jmf}(\eta)$; $\tilde{u}(x, z), \tilde{v}(x, z)$ are harmonical functions of the real variables $x$ and $z$. In the work by Vekua (1960) it was shown that relation (3) presents all possible solutions of Eq. (1). Thus, the problem is reduced to the choice of a particular function $f(\eta)$, consistent with the adopted symmetry of the problem and boundary conditions.

As is mentioned in the Introduction, we consider the twodimensional plasma sheet in the region $-\infty<x \leq x_{b}$, $-\infty<z<+\infty$. On the upper and lower boundaries uniform boundary conditions are set

$B_{x}=-\frac{\partial A}{\partial z^{*}} \rightarrow \pm B_{\infty}, z \rightarrow \pm \infty$.

On the right boundary $(x \rightarrow-\infty)$ the solution is required to transfer to that of Harris (1962), with $A(-\infty, 0)=0$ and $n(-\infty, 0)=n_{0}$. As distinct from the Kan treatment when it was reasonable to give in the centre of the sheet at the inner boundary the values of the magnetic field intensity and plasma density, in the case under consideration, it is reasonable to give the maximum information on the magnetic field, while the plasma density distribution along the sheet has to be obtained from the solution of the corresponding nonlinear, self-consistent problem. We introduce into the boundary conditions the following information on the field of the inner magnetosphere: at the point $x=x_{b}$ we give not only the value of $B_{z}\left(x_{b}, 0\right)$ but also $\left.\frac{\partial}{\partial x} B_{z}(x, 0)\right|_{x=x_{b}}$ and suppose them to be equal to the corresponding values of the field of the internal sources. Having set the boundary conditions, in the next section we will determine the appropriate form of the function $f(\eta)$.

\section{Stationary configurations of the nonuniform plasma sheet set by different conditions on the near-Earth and far tail boundaries}

Manankova et al. (2000a, b) classified all known exact solutions of Eq. (1). The simplest one is the solution of Harris (1962)

$f(\eta)=\exp \eta$,

which is uniform along the $x$-axes' plasma sheet, with $x$ varying from $-\infty$ to $+\infty$. If $f(\eta)$ has the form

$f(\eta)=\exp \left\{\eta+\frac{\lambda}{\eta-i \alpha}\right\}$, 
then we obtain the solution of Kan (1973), which contains the major term that determines the decline of the plasma density and magnetic field $B_{z}$-component down the tail.

If we take

$f(\eta)=\tilde{\gamma}\left\{\tilde{C}+\exp \left[\eta+\frac{\lambda}{\eta-i \alpha}\right]\right\}$

we have the solution of Manankova et al. (2000a, b), which similar to Eq. (5) describes the decline of $n$ and $B_{z}$ when moving tailward and, in addition, for $\tilde{C} \neq 0$ yields a nonuniform, spatially oscillating branch of the solution, i.e. a set of filaments. This branch is not treated in the present work.

For the boundary conditions set in the manner discussed in the previous section, the function $f(\eta)$ should be taken in the form that includes one more term of expansion compared to Eq. (5)

$f(\eta)=\exp \{\eta+\varphi(\eta)\}, \varphi(\eta)=\frac{\lambda}{\eta-i \alpha}+\frac{\lambda_{1}}{(\eta-i \alpha)^{3}}$

where $\lambda$ and $\lambda_{1}$ are constant and determined from the boundary conditions. Since we suggest for the left boundary: $x=x_{b} \neq 0$, we can set $\alpha=0$. Then, with the use of Eqs. (2) and (3) the expression for $n(x, z)$ and $A(x, z)$ can be written in the following form:

$$
\begin{aligned}
& a A(x, z)=\ln \frac{\left(1+u_{z}\right)^{2}+u_{x}^{2}}{c h^{2}[z+u(x, z)]}, \quad a=\frac{2 m}{B_{\infty}}=\frac{q \beta}{\Theta} \\
& n(x, z)=n_{0} \exp a A(x, z), u(x, z) \equiv \operatorname{Re} \varphi(\eta) \\
& \frac{B_{x}(x, z)}{B_{\infty}}=\left(1+u_{z}\right) t h(z+u) \\
& -\frac{\left(1+u_{z}\right) u_{z z}+u_{x} u_{x z}}{\left(1+u_{z}\right)^{2}+u_{x}^{2}} \\
& \frac{B_{z}(x, z)}{B_{\infty}}=-u_{x} t h(z+u)+\frac{\left(1+u_{z}\right) u_{z x}+u_{x} u_{x x}}{\left(1+u_{z}\right)^{2}+u_{x}^{2}} \\
& u(x, z)=\frac{\lambda z}{D}+\frac{\lambda_{1}\left(z^{3}-3 z x^{2}\right)}{D^{3}}, D=z^{2}+x^{2} \\
& u_{x}=\frac{\partial}{\partial x} u(x, z)=-\frac{2 \lambda z x}{D^{2}}-\frac{12 \lambda_{1} z x\left(z^{2}-x^{2}\right)}{D^{4}} \\
& u_{z}=\frac{\partial}{\partial z} u(x, z)=\frac{\lambda\left(x^{2}-z^{2}\right)}{D^{2}} \\
& \quad+\frac{3 \lambda_{1}\left(-x^{4}-z^{4}+6 x^{2} z^{2}\right)}{D^{4}} \\
& u_{x x}=-\frac{12 \lambda_{1} z\left(z^{4}+5 x^{4}-10 x^{2} z^{2}\right)}{D^{5}} . \frac{-2 \lambda z\left(z^{2}-3 x^{2}\right)}{D^{3}} \\
& u_{z x}=\frac{\partial^{2} u(z, x)}{\partial x \partial z}=\frac{2 \lambda x\left(-x^{2}+3 z^{2}\right)}{D^{3}} \\
& +\frac{12 \lambda_{1} x\left(x^{4}+5 z^{4}-10 x^{2} z^{2}\right)}{D^{5}}
\end{aligned}
$$

Let us consider more in detail by determining of $\lambda$ and $\lambda_{1}$. From Eq. (7) the $B_{z}$-component of the magnetic field in the centre of the sheet is given by the formula

$\frac{B_{z}(x, 0)}{B_{\infty}}=\frac{u_{z x}(x, 0)}{1+u_{z}(x, 0)}$,

where

$u_{z}(x, 0)=\frac{\lambda}{x^{2}}-\frac{3 \lambda_{1}}{x^{4}}, u_{z x}(x, 0)=\frac{-2 \lambda}{x^{3}}+\frac{12 \lambda_{1}}{x^{5}}$.

It is convenient to change $\lambda$ and $\lambda_{1}$ for $y=\lambda / x_{b}^{2}$ and $y_{1}=$ $\lambda_{1} / x_{b}^{4}$. With the use of the boundary conditions one obtains the following system of two equations for $y$ and $y_{1}$

$$
\begin{aligned}
& \frac{B_{z}\left(x_{b}, 0\right)}{B_{\infty}} \cdot x_{b}=\frac{-2 y+12 y_{1}}{1+y-3 y_{1}} \equiv \gamma \\
& \left.\frac{\partial}{\partial x}\left(\frac{B_{z}(x, 0)}{B_{\infty}}\right)\right|_{x=x_{b}} \cdot x_{b}^{2}=\frac{6 y-60 y_{1}}{1+y-3 y_{1}}-\gamma^{2} \equiv \gamma_{1} .
\end{aligned}
$$

The solution of this system is

$y=\frac{-6(C+5) \gamma}{[3 \gamma C+21 \gamma+24]}, \quad y_{1}=\frac{-\gamma(C+3)}{[3 \gamma C+21 \gamma+24]}$,

where $C=\left(\gamma_{1}+\gamma^{2}\right) / \gamma$. Further in the numerical calculations the magnetic field of the inner magnetosphere is taken as that of a two-dimensional dipole: $B_{z d}(x, 0)=M / x^{2}$. In this case there can be found a simple relation between $\gamma$ and $\gamma_{1}$ (cf. Eq. 8):

$\frac{\gamma_{1}}{\gamma}=\left.\left[\frac{\frac{\partial}{\partial x} B_{z}(x, 0) \cdot x}{B_{z}(x, 0)}\right]\right|_{x=x_{b}}=-2, \quad C=\gamma-2$

and from Eq. (9) we have for $y$ and $y_{1}$ :

$y=\frac{-6 \gamma(\gamma+3)}{\left(3 \gamma^{2}+15 \gamma+24\right)}, \quad y_{1}=\frac{-\gamma(\gamma+1)}{\left[3 \gamma^{2}+15 \gamma+24\right]}$.

In the consideration that has been performed above the typical scale of the problem is the characteristic thickness of the plasma sheet $L=1 / \mathrm{m}$. However, in the treatment of the quasi-stationary evolution of the system, which is the subject of the following sections, this parameter will be variable. Thus, it is more convenient to transfer to the Earth's radius $R_{E}$ as a scale size of the problem. Having denoted $\bar{x}=x^{*} / R_{E}, \bar{z}=z^{*} / R_{E}, \bar{m}=m R_{E}, \bar{\lambda}=\lambda / \bar{m}^{2}$, $\bar{\lambda}_{1}=\lambda_{1} / \bar{m}^{4}$, we can rewrite some of relations (7) as

$$
\begin{gathered}
u(\bar{x}, \bar{z})=\bar{m}\left\{\frac{\bar{\lambda} \bar{z}}{\bar{D}}+\frac{\bar{\lambda}_{1}\left(\bar{z}^{3}-3 \bar{z} \bar{x}^{2}\right)}{\bar{D}^{3}}\right\}, \\
\bar{D}=\bar{x}^{2}+\bar{z}^{2} \\
u_{x}(\bar{x}, \bar{z})=\frac{-2 \bar{\lambda} \bar{z} \bar{x}}{\bar{D}^{2}}-\frac{12 \bar{\lambda}_{1} \bar{z} \bar{x}\left(\bar{z}^{2}-\bar{x}^{2}\right)}{\bar{D}^{4}}
\end{gathered}
$$




$$
\begin{aligned}
u_{z}(\bar{x}, \bar{z}) & =\frac{\bar{\lambda}\left(\bar{x}^{2}-\bar{z}^{2}\right)}{\bar{D}^{2}} \\
+ & \frac{3 \bar{\lambda}_{1}\left(-\bar{x}^{4}-\bar{z}^{4}+6 \bar{x}^{2} \bar{z}^{2}\right)}{\bar{D}^{4}} \\
z+u(x, z) & =\bar{m}\left\{\bar{z}+\frac{\bar{\lambda} \bar{z}}{\bar{D}}+\frac{\bar{\lambda}_{1}\left(\bar{z}^{3}-3 \bar{z} \bar{x}^{2}\right)}{\bar{D}^{3}}\right\} .
\end{aligned}
$$

The quantities $\bar{\lambda}=\bar{x}_{b}^{2} y$ and $\bar{\lambda}_{1}=\bar{x}_{b}^{4} y_{1}$ can be determined with the use of Eq. (10).

\section{Parameters of the solution. Physical mechanism of the thin current sheet formation in the near-Earth magnetotail}

As was mentioned above, when moving tailward from the near-Earth boundary $x=x_{b}$, the solution obtained tends to that of Harris. The latter is uniform along the $x$ current sheet, which is characterized by five parameters with the two relations imposed, that is only three of the parameters are independent. The five parameters are

$B_{\infty}, n_{0}, \beta=V / c, \Theta=k T, m=1 / L$.

The two relations are

$B_{\infty}^{2}=16 \pi n_{0} \Theta, m^{2}=4 \pi n_{0} \beta^{2} q^{2} / \Theta$.

In modeling a quasi-stationary evolution of the current sheet we must stipulate which of parameters in Eq. (12a) will be altered and what their variation should be to reproduce correctly the current sheet perturbations at different stages of magnetospheric disturbances. A problem concerning stationary configurations of an nonuniform, current-carrying, twodimensional plasma sheet with boundary conditions given on cross-sheet profiles at the foot of the sheet and infinity is considered, with the aim of using its solution for the description of the interaction of the two current systems: one is related to the rather stationary inner magnetosphere and the other is that of the dynamical magnetospheric tail.

The solution is determined by different combinations of parameters in Eq. (12a) as well as by $\bar{x}_{b}$ (the near-Earth boundary location) and $B_{z d}\left(\bar{x}_{b}, 0\right)$, which, in turn, depends on $\bar{x}_{0}$ - the distance involved in the normalization of the 2D dipolar field over a 3-D field. In the present work we take $\bar{x}_{0}=-10$ and $B_{z d}(x, 0)=3 \cdot 10^{3} / \bar{x}^{2}(n T)$, according to the magnetospheric magnetic field model of Tsyganenko (Tsyganenko, 1989). The combinations of the parameters that entered the solution are

$\gamma=B_{z d}\left(\bar{x}_{b}, 0\right) \cdot \bar{x}_{b} \cdot m R_{E} / B_{\infty}$,

$m / B_{\infty}=\frac{a}{2}=q \beta / 2 \Theta$.

In addition, the solution depends on $m, \bar{x}_{b}, n_{0}, B_{\infty}$. Thus, five independent parameters determine the solution: three independent parameters from (12a, b), $\bar{x}_{b}$ and $\bar{x}_{0}$.
Now we consider the quasi-stationary dynamics of the plasma sheet by altering some of these parameters in four special cases.

(a) Let the disturbance of the sheet, caused by $B_{\infty}$ (or $n_{0}$ ), increase under invariable temperature $\Theta$, current velocity $\beta$ and the location of the left boundary $\bar{x}=\bar{x}_{b}$. Then $m / B_{\infty}=$ const, $\gamma=$ const. This means that $m$ grows proportionally to $B_{\infty}$, leading to a contraction of the plasma sheet.

(b) Let the temperature $\Theta$ increase under invariable $\bar{x}_{b}, \beta$ and $n_{0}$. Then $m \sim \Theta^{-1 / 2}, B_{\infty} \sim \Theta^{1 / 2}, m / B_{\infty} \sim \Theta^{-1}$ and $\gamma \sim \Theta^{-1}$. These relations indicate that by changing the temperature we inevitably change some of the other parameters characterizing the plasma sheet (this is also true for point (a)). Thus, $\Theta^{(0)}, \gamma^{(0)}, m^{(0)}$, $B_{\infty}^{(0)}$ refer to the undisturbed state of the plasma sheet, and $\gamma^{(1)}, m^{(1)}, B_{\infty}^{(1)}$ relate to the altered temperature $\Theta=\Theta^{(1)}$, then $\gamma^{(1)}=\gamma^{(0)} \Theta^{(0)} / \Theta^{(1)}, m^{(1)}=m^{(0)}$. $\left(\Theta^{(0)} / \Theta^{(1)}\right)^{1 / 2}, B_{\infty}^{(1)}=B_{\infty}^{(0)} \cdot\left(\Theta^{(1)} / \Theta^{(0)}\right)^{1 / 2}$, etc.

(c) Let the disturbance be connected to the acceleration of the charged particles in the neutral sheet, i.e. with the growth of $\beta$ under $n_{0}$, $\Theta$, with $\bar{x}_{b}$ being constant. Then $\gamma \sim \beta, m \sim \beta, a=2 m / B_{\infty} \sim \beta$. Therefore, similar to point (b) we obtain $\beta^{(1)} / \beta^{(0)}=a^{(1)} / a^{(0)}=$ $\gamma^{(1)} / \gamma^{(0)}=m^{(1)} / m^{(0)}$.

(d) The increase of $B_{\infty}$, which is related to energy accumulation in the magnetospheric tail, typically displaces the location of the left boundary $\bar{x}=\left|\bar{x}_{b}\right|$ closer to the Earth. This results in $\left|\bar{x}_{b}\right|$ decrease and variation of the parameter $\gamma$ according to the relation $\gamma_{1} / \gamma_{0}=$ $x_{b}^{(0)} / x_{b}^{(1)}$. As will be further demonstrated, this variation is of principal importance, for it controls reconfiguration of the magnetic fields and currents after $\gamma$ reaching the critical value of $\gamma_{c r}$.

Though the special cases considered do not cover all possible quasi-dynamical variations of the plasma sheet, they clearly suggest that the parameters governing the state of the sheet change self-consistently in the process of its quasi-stationary evolution.

To complete this section we draw the reader's attention to thin current sheets originating in the near-Earth magnetotail at the growth phase of a substorm. The mechanism of their formation has been discussed in literature for the last two decades. Among the theoretical studies devoted to this problem the most complete are those by Schindler and colleagues (e.g. Schindler et al., 1993). The authors consider the quasi-stationary evolution of the magnetotail current in the framework of the linearized Grad-Shafranov equation with the boundary conditions of either of the two types discussed in the Introduction. The Harris solution is taken as an initial unperturbed state of the current sheet. The parameters of the Harris solution are kept invariable in the course of the current sheet evolution, with the latter being entirely governed 

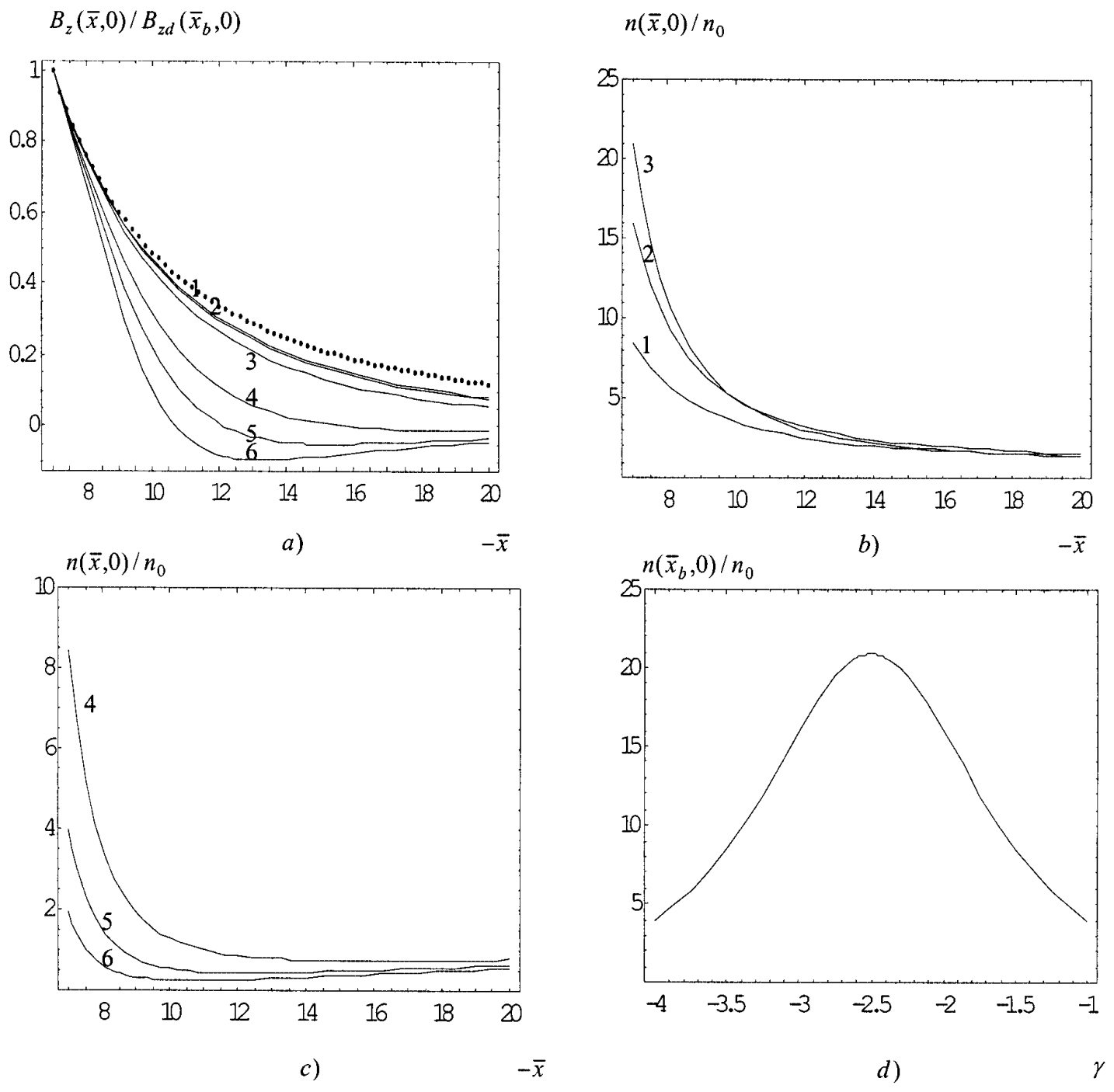

Fig. 1. (a) Variation of the dimensionless field component $B_{z}(\bar{x}, 0) / B_{z d}\left(\bar{x}_{b}, 0\right)$ along the sheet versus the dimensionless distance $\bar{x}$. For comparison, the variation $B_{z d}(\bar{x}, 0) / B_{z d}\left(\bar{x}_{b}, 0\right)$ is shown by a dotted line. (b), (c) Variation of the dimensionless plasma density (current density) $n(\bar{x}, 0) / n_{0}=j(\bar{x}, 0) / j_{0}$. (d) The plasma density $n\left(\bar{x}_{b}, 0\right) / n_{0}$ under $\bar{x}=\bar{x}_{b}=-7$ as a function $\gamma$. Numbers at curves correspond: $\gamma_{1}=-1.5, \gamma_{2}=-2.0, \gamma_{3}=-2.5, \gamma_{4}=-3.5, \gamma_{5}=-4, \gamma_{6}=-4.5$.

by quasi-static changes in the boundary conditions. Thin current sheet formation as a slow process driven by boundary deformations is also considered in a more recent work by Becker et al. (2001 and references therein). In comparing these treatments with the present study, it is clear that the underlying physical mechanism suggested here is different: the current sheet with the parameters that vary self-consistently in the process of its evolution adjusts to the given boundary conditions.

Among the four special cases considered in this section we point out case (d), in which both the current sheet parameters and boundary conditions (through the value of $x_{b}$ ) are variable. However, we stress again that their variations are self-consistent. The possibility to treat them independently, for example, as additive contributors, needs special argumentation.

\section{Specialities and applications of the solution obtained}

The most prominent dynamical processes in the magnetosphere are known to proceed in the region of transition from dipolar to stretched magnetic field configuration, where the scale sizes along the $x$ - and $z$-coordinates are of the same order: $O\left(L_{x}\right)=O\left(L_{z}\right)$; here, $O$ is the standard order symbol, $L_{z}, L_{x}$ denote characteristic lengths for variation in the $z$ and $x$-direction. Therefore, for this region we cannot apply the model problem solutions in which the so-called "tail-like approximation" $L_{x} \gg L_{z}$ (Schindler, 1972; Birn et al., 1975) is suggested. The solution obtained in the present paper has a simple form (especially in the centre of the current sheet $(z=0))$ and is not restricted by any special requirements: the solution obtained is the exact analytical solution. In this section we use it to follow the main features of the magnetic field and current quasi-static evolution. 


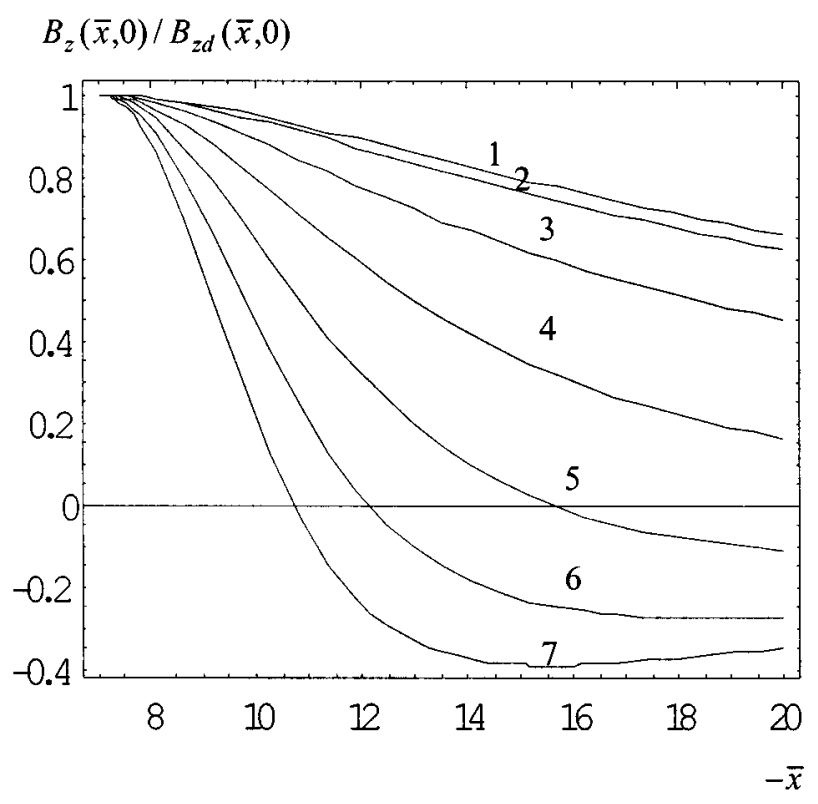

Fig. 2. Variation of the dimensionless field component $B_{z}(\bar{x}, 0) / B_{z d}(\bar{x}, 0)$ along the sheet. Numbers at the curves correspond: $\gamma_{1}=-1.5, \gamma_{2}=-2.0, \gamma_{3}=-2.5, \gamma_{4}=-3.0, \gamma_{5}=-3.5$, $\gamma_{6}=-4.0, \gamma_{7}=-4.5$.

According to Eqs. (7) and (10) the magnetic field $B_{z}(\bar{x}, 0)$ is given by the relation

$B_{z}(\bar{x}, 0)=\frac{B_{\infty}}{\bar{m} \bar{x}_{b}} \cdot \frac{-2 y\left(\frac{\bar{x}_{b}}{\bar{x}}\right)^{3}+12 y_{1}\left(\frac{\bar{x}_{b}}{\bar{x}}\right)^{5}}{1+y\left(\frac{\bar{x}_{b}}{\bar{x}}\right)^{2}-3 y_{1}\left(\frac{\bar{x}_{b}}{\bar{x}}\right)^{4}}$.

If we take into account formula (13), then $B_{\infty} /\left(\bar{m} \cdot \bar{x}_{b}\right)=$ $B_{z d}\left(\bar{x}_{b}, 0\right) / \gamma$. As is seen from Eq. (14), the peak value of $B_{z}(\bar{x}, 0)$ is determined by $B_{z d}\left(\bar{x}_{b}, 0\right)$, i.e. depends on the left boundary location, while the $B_{z}(\bar{x}, 0)$ distribution along the sheet is mostly controlled by the value of $\gamma$. The plasma density distribution, which, in our consideration, coincides with that of the current density, can be described as follows

$n(\bar{x}, 0) / n_{0}=\left[1+y\left(\frac{\bar{x}_{b}}{\bar{x}}\right)^{2}-3 y_{1}\left(\frac{\bar{x}_{b}}{\bar{x}}\right)^{4}\right]^{2}=j(\bar{x}, 0) / j_{0}$,

where $j_{0}=j(-\infty, 0)$.

It is interesting to note that expression (14), depending on the value of $\gamma$, indicates either a monotonous decline of $B_{z}(\bar{x}, 0)$ down the tail or a non-monotonous distribution, having a point $\bar{x}_{R}$ such as $B_{z}\left(\bar{x}_{R}, 0\right)=0$. This point location can be found as

$\bar{x}_{R}^{2} / \bar{x}_{b}^{2}=(\gamma+1) /(\gamma+3)$.

From formulas (14)-(16) it follows that for $\gamma>-3$ we have a monotonous decline of $B_{z}(\bar{x}, 0)$ and $n(\bar{x}, 0)$ (Fig. 1a, b), while the case $\gamma<-3$ corresponds to the non-monotonous profile with a minimum in the region of negative $B_{z}(\bar{x}, 0)$ (Fig. 1a). Note that the configuration with the $x_{R}$-point results in the non-monotonous profile $n(x, 0)$, namely the profile with a minimum of the current density (Fig. 1c). These features of the magnetic field and current density distributions can be relevant to the substorm development, for it is known that the expansion phase instability is related to the region of local decrease in the cross-tail current and to that of small $B_{z}$ values.

By using formulas (14)-(15), one can estimate the typical extent of the current sheet perturbation $x_{l}$ caused by the left boundary condition. In the case $\gamma<-3$ it can be roughly taken as $x_{l} \approx x_{R}$, then from formula (16) we obtain $\left(x_{l} / x_{b}\right)^{2}=(\gamma+1) /(\gamma+3)$. One more relation following from the solution seems informative

$$
\frac{B_{z}(\bar{x}, 0)}{B_{z d}(\bar{x}, 0)}=\frac{-2 y \frac{\bar{x}_{b}}{\bar{x}}+12 y_{1}\left(\frac{\bar{x}_{b}}{\bar{x}}\right)^{3}}{\gamma\left[1+y\left(\frac{\bar{x}_{b}}{\bar{x}}\right)^{2}-3 y_{1}\left(\frac{\bar{x}_{b}}{\bar{x}}\right)^{4}\right]} .
$$

This relation shows how far from the boundary $x=x_{b}$ down the tail the dipolar magnetic field remains essential. From Fig. 2 one can see that the larger the value of $|\gamma|$ or, in other words, the more intense the cross-tail current is, the narrower the region where the dipolar magnetic field is not compensated for by the field of the external source.

Let us rewrite Eq. (17) in a more convenient form

$$
\frac{B_{z}(\bar{x}, 0)}{B_{z d}\left(\bar{x}_{b}, 0\right)}=\frac{B_{z d}(\bar{x}, 0)}{B_{z d}\left(\bar{x}_{b}, 0\right)} \cdot \frac{-2 y \frac{\bar{x}_{b}}{\bar{x}}+12 y_{1}\left(\frac{\bar{x}_{b}}{\bar{x}}\right)^{3}}{\gamma\left[1+y\left(\frac{\bar{x}_{b}}{\bar{x}}\right)^{2}-3 y_{1}\left(\frac{\bar{x}_{b}}{\bar{x}}\right)^{4}\right]} .
$$

As is seen from Eq. (18), Fig. 1a (the dotted line) and Fig. 2, the ratio $B_{z}(\bar{x}, 0) / B_{z d}\left(\bar{x}_{b}, 0\right)$ becomes of the order of $O(\varepsilon)$, with $\varepsilon$ being a small parameter, at such distances where it was of the order of $O(1)$ in the absence of the current sheet: $B_{z d}(\bar{x}, 0) / B_{z d}\left(\bar{x}_{b}, 0\right)=O(1)$. In the region where in the absence of the current sheet the ratio $B_{z d}(\bar{x}, 0) / B_{z d}\left(\bar{x}_{b}, 0\right)$ was of the order of $O(\varepsilon)$, it will be of the order of $O\left(\varepsilon^{2}\right)$, i.e. in this region the left boundary condition does not affect the current sheet. Here, the sheet can be considered as a uniform sheet, with the accuracy of $O\left(\varepsilon^{2}\right)$. Of course, all this is valid only for uniform boundary conditions along the sheet. If the latter slowly varies down the tail, this will cause the sheet's nonuniformity $B_{z}(x, 0) / B_{\infty}=O(\varepsilon)$. But this mechanism is principally different from what we suggest.

It is interesting to compare the consideration performed in this section with the results of the studies that treat the same problem under the "tail-like approximation" (Wang and Bhattacharjee, 1999). The authors consider $L_{z} / L_{x}=O(\varepsilon)$, neglect the terms of the order of $O\left(\varepsilon^{2}\right)$ and reduce the twodimensional Grad-Shafranov equation to a one-dimensional equation that has a solution of the form $A(z, \varepsilon x)$, with the transition region, where $L_{x} / L_{z}=O(1)$ is excluded from the treatment. We note that in the region where $L_{z} / L_{x}=O(\varepsilon)$, the ratio $B_{z}(x, 0) / B_{\infty}$ is also of the order of $O(\varepsilon)$. According to our results, at distances where the "tail-like approximation" remains true the perturbation of the order of $O(\varepsilon)$ is 


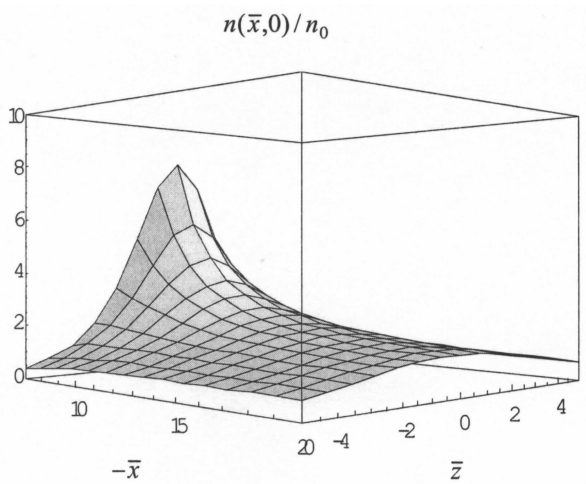

a)

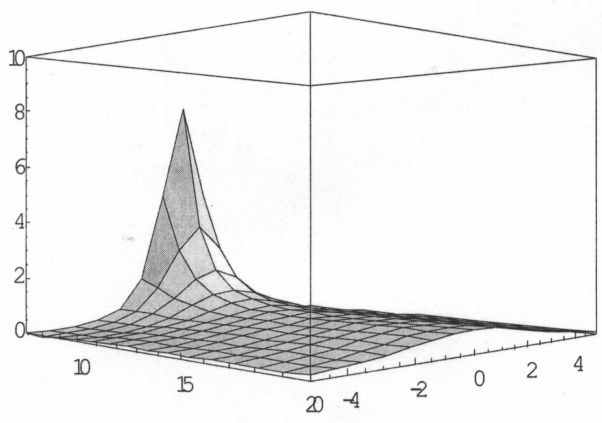

c)

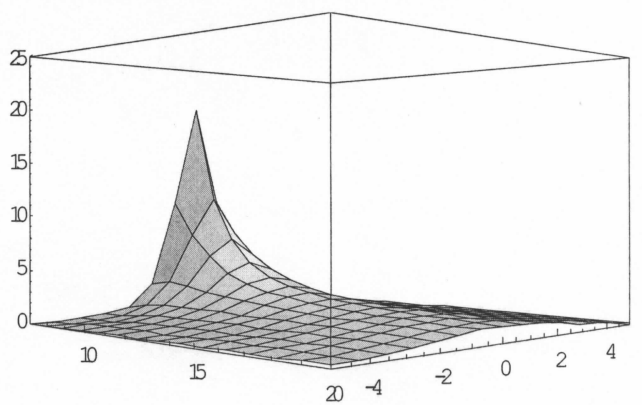

b)

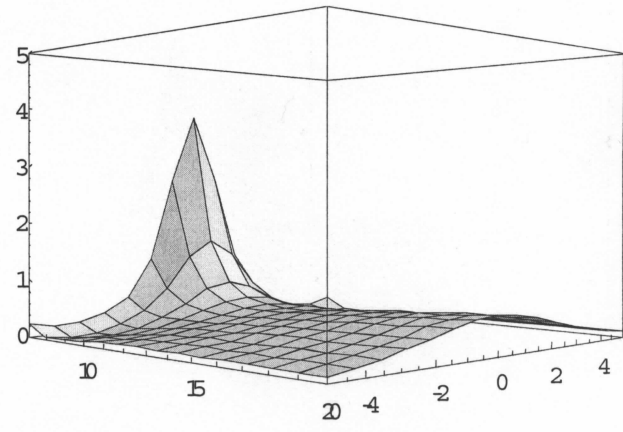

d)

Fig. 3. Dimensionless plasma density (current density) relief: $n(\bar{x}, \bar{z}) / n_{0}=j(\bar{x}, \bar{z}) / j_{0}$. The values $\bar{x}_{b}$ and $B_{\infty}$ are fixed: $\bar{x}_{b}=-7$, $B_{\infty}=\frac{3 \cdot 10^{2}}{7}$ (nT). (a) $\gamma=-1.5, \bar{m}=0.15$; (b) $\gamma=-2.5, \bar{m}=0.25 ;$ (c) $\gamma=-3.5, \bar{m}=0.35, \bar{x}_{R}=-15,6$; (d) $\gamma=-4, \bar{m}=0.4$, $\bar{x}_{R}=-12.1$. Note the difference in vertical scales in the upper and lower panels.

already "compensated" by the current sheet, i.e. only terms $O\left(\varepsilon^{2}\right)$ are non-zero. These terms cannot be found in the framework of a one-dimensional Grad-Shafranov equation.

Now we consider more in detail the principal features of the obtained solution.

1. For $\gamma \leq-3$ at the point $\bar{x}=\bar{x}_{R}$ given by Eq. (16) we have $B_{z}\left(\bar{x}_{R}, 0\right)=0, B_{x}\left(\bar{x}_{R}, 0\right)=0$ and $B_{z}\left(\bar{x}_{R}, 0\right) / B_{x}\left(\bar{x}_{R}, 0\right)=0$, when $\left|\bar{x}_{R}\right| \rightarrow \infty\left(\gamma=\gamma_{c r}\right)$, and $B_{z}\left(\bar{x}_{R}, 0\right) / B_{x}\left(\bar{x}_{R}, 0\right) \neq 0$, when $\left|\bar{x}_{R}\right| \neq \infty(\gamma \neq$ $\left.\gamma_{c r}\right)$. The ratio $B_{z}\left(\bar{x}_{R}, 0\right) / B_{x}\left(\bar{x}_{R}, 0\right)$ is a slope of the magnetic field line at $\bar{x}=\bar{x}_{R}$. Thus, we can see that the magnetic field lines are tangential only at the point $\left|\bar{x}_{R}\right|=\infty$, when $\gamma=\gamma_{c r}=-3$. Note that we examine the same (non-filamentary) branch of the solution of the Grad-Shafranov equation versus $\gamma$. The value $\gamma=\gamma_{c r}=-3$ is not referred to as the origin of a bifurcation point: the filamentary branch of the solution exists independently.

2. From Eq. (15) it follows that under $\gamma>-3$ the current sheet adjusts to the left (near-Earth) boundary condition by increasing both plasma and current density in the transition region, with $|\gamma|$ increasing as well (Fig. 1b, d). As a result, in the region adjacent to the left boundary the density of the current is by an order of magnitude larger and the sheet is essentially thinner than it is farther down the tail (Fig. 3a, b). At this stage of the current density growth, the displacement of the contours $n(\bar{x}, \bar{z}) / n_{0}=$ const proceeds tailward (Fig. 4a, b). Thus, one can see that it is possible to describe the formation of very thin, intense current sheets in the transition region in terms of the simple exact solution of the Grad-Shafranov equation. For $\gamma<-3$ the current sheet adjusts to the left boundary condition by decreasing the plasma and current densities in the transition region, which is accompanied by a reconfiguration of the magnetic field (Figs. 1a, c, d and 3c, d). At this stage of the current density reduction, in the adjacent to the left boundary region, the displacement of the contours $n(\bar{x}, \bar{z}) / n_{0}=$ const that cross the $x$-axes at $x>x_{R}$ is toward the Earth, while those that cross the $x$-axes at $x<x_{R}$ are tailward (Fig. $4 \mathrm{c}, \mathrm{d}$ ).

The physical mechanism of the magnetic field, plasma and current density redistribution discussed above can be realized as follows. The current-carrying plasma sheet is a spatially confined congestion of particles (Vlasov, 1961) held by Ampere force. Conglomeration of plasma elements can be considered to be due to the attraction of the elementary parallel "current threads" which form the cross-tail current (Braginsky, 1957). Under uniform boundary conditions such a system with 


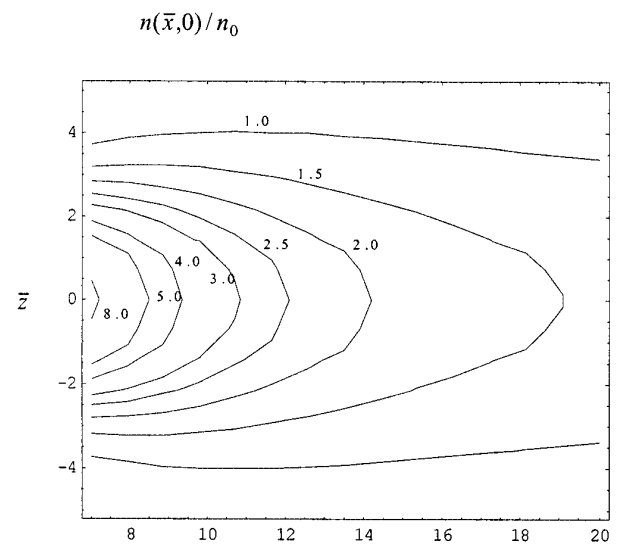

a)

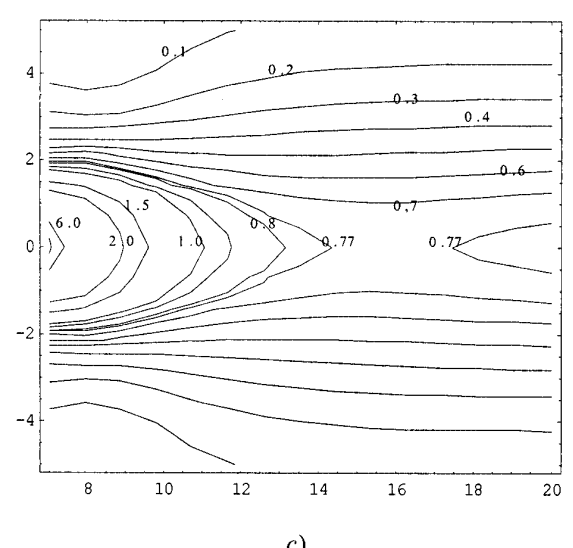

c)

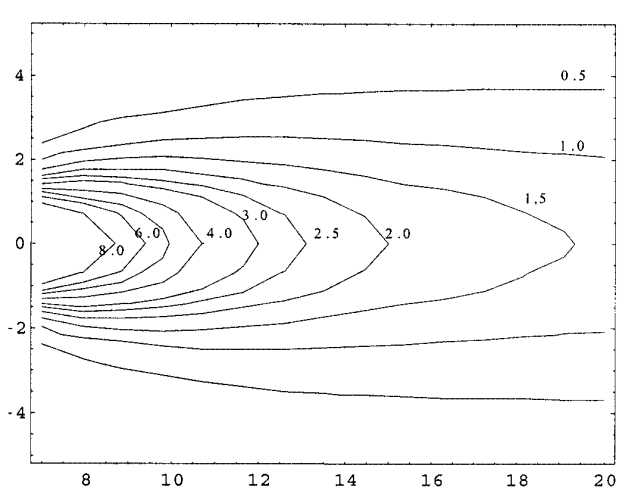

b)

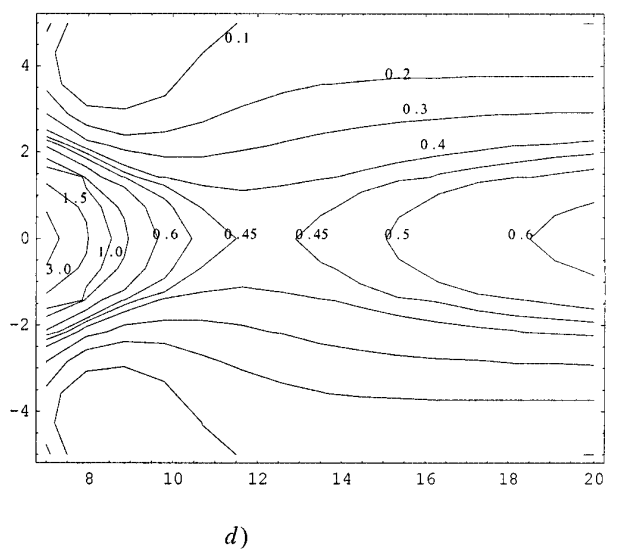

d)

Fig. 4. Isolines of the plasma density (current density): $n(\bar{x}, \bar{z}) / n_{0}=j(\bar{x}, \bar{z}) / j_{0}$. Number of the curves corresponds to the value of the dimensionless plasma density (current density). The value $\bar{x}_{b}, B_{\infty}, \gamma, \bar{m}$ as in Fig. 3.

equal probability can stay either as a homogeneous or filamentary configuration, with the scale size of the filaments being determined as (Vlasov, 1961):

$l_{j}^{2}=\frac{\Theta}{m \omega_{j}^{2}}, \quad \omega_{j}^{2}=\frac{4 \pi\left(\frac{j}{c}\right)^{2}}{\rho}$,

where $\omega_{j}$ is the eigenfrequency, $\Theta$ is the characteristic energy, $\rho$ is the plasma mass density, and $j$ is the current density. Equation (19), known as a pinch-effect condition, can be treated as a state equation relating the linear scales of a plasmoid to the temperature, mass density and magnetic field. In these terms the formation of a narrow channel with a minimum of the current density and negative $B_{z}$ values (further referred to as "a neck") described by our solution can be interpreted as a result of "competition" between the two distinct regions of the cross-tail current: the one adjacent to the left boundary under $\gamma$, having reached $\gamma_{c r}$, and the other infinitely stretched down the tail uniform sheet by an order of magnitude smaller current density, either of which tends to draw the current elements from the region of the "neck" development.

3. The contours $n(x, z)=$ const, with the governing parameters being fixed, are also the isocontours of the vector potential $A(x, z)=$ const. However, unlike the model solutions with a quasi-stationary altering of the boundary conditions, our treatment of dynamics of the density contours does not reproduce identically the dynamics of the magnetic field lines. Indeed, according to Eq. (2) $n(x, z)=n(A(x, z), a)$, the parameter $a=2 m / B_{\infty}$, controlling the current sheet far down the tail, can change in the process of quasi-static evolution (see also Sect. 4). The difference in behaviour of the density and vector potential isocontours can also be followed from the functional relation between them. Let $a_{0}$ be the value of the $a$ parameter over which the vector potential is normalized. Then, from Eqs. (2) and (13) we have

$$
a_{0} A(x, z)=\frac{a_{0}}{a} \ln \frac{n(x, z)}{n_{0}}=\frac{\gamma_{0}}{\gamma} \ln \frac{n(x, z)}{n_{0}} .
$$


$a_{0} A(\bar{x}, \bar{z})$
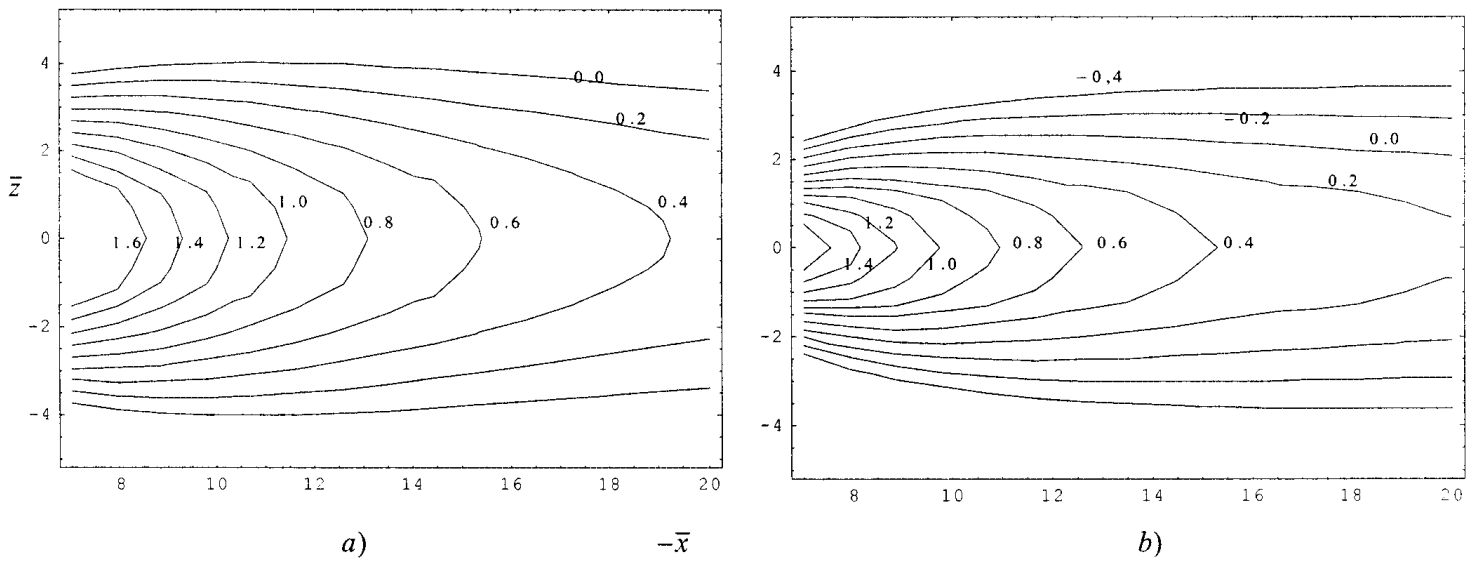

b)

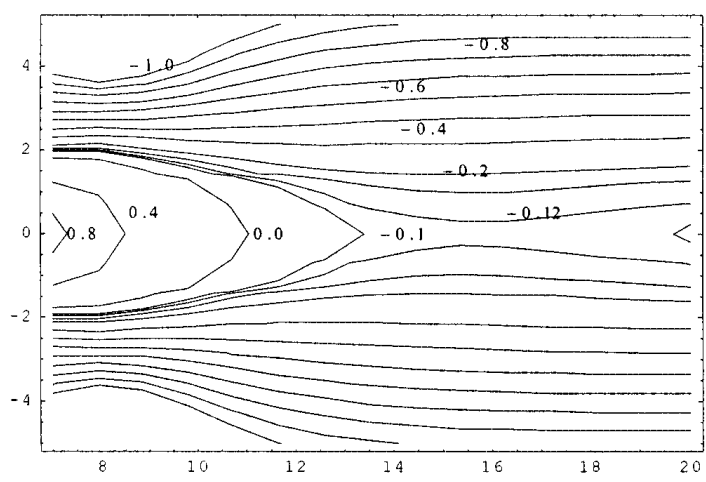

c)

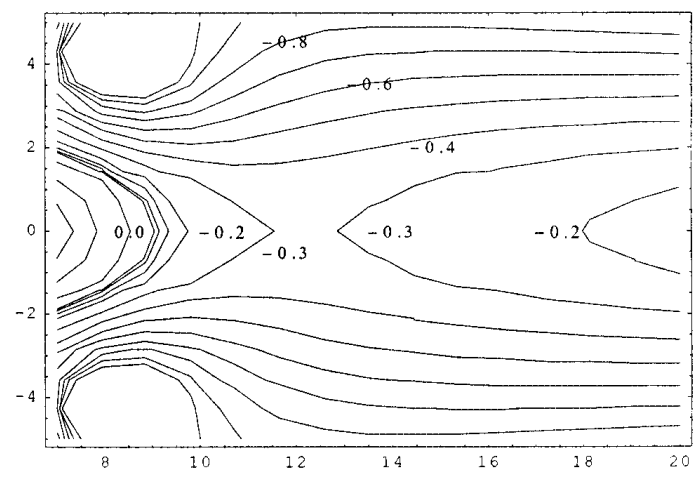

d)

Fig. 5. Isolines of the dimensionless vector potential $a_{0} A(x, z)=\frac{\gamma_{0}}{\gamma} \ln \frac{n(x, z)}{n_{0}}$. The value $\bar{x}_{b}, B_{\infty}, \gamma, \bar{m}$ as in Fig. $3, a_{0}, \gamma_{0}-$ the value of parameter $a=2 \bar{m} / R_{E} B_{\infty}$, and the value of parameter $\gamma$ (13) as at Fig. 3a. Number of the curves corresponds to the value of vector potential $a_{0} A(x, z)$.

Since in a perfectly conductive plasma the frozen-in condition is held, Figs. 5a, b, c, d indicate not only redistribution of the magnetic field lines, but also plasma displacement with the governing parameters being altered.

4. For understanding the underlying mechanism of development of the strongly nonuniform current sheet in the near-Earth region, it is important to remember that we have not used linearization of the Grad-Shafranov equation, i.e. its reduction to the Shrödinger equation. In other words, the solution obtained is an exact solution of the nonlinear problem. With this respect there are a few points to be clarified. In the most interesting transition region we have $j(x, z) / j_{0}=O\left(\varepsilon^{-1}\right)$, with $\varepsilon$ being a small parameter, $j_{0}$ being the current density at the centre of the sheet far down the tail. Having taken into account the relation $j(x, z) / j_{0}=\exp a A(x, z)$, one can see that in this case we cannot use linearization of the right-hand-side of Eq. (1), for the resulting expansion

$$
\begin{aligned}
\exp a A(x, z) & =\exp a\left[A_{0}(z)+A_{1}(x, z)\right] \\
& \simeq \frac{1}{c h^{2}(z)}\left[1+a A_{1}(x, z)+O\left(\varepsilon^{2}\right)\right]
\end{aligned}
$$

in which $A_{0}(z)$ is the part of the vector potential related to the Harris sheet, only describes the effects of the order of $O(\varepsilon)$. Another aspect which has already been discussed above, is that the pinch-effect and the sheet filamentation are intrinsically nonlinear processes. The treatments under a linear approximation can only give a sinusoidal variation of the current distribution, not the filaments formation (Trubnikov, 2002). 
5. Only one type of solution of Eq. (1) is treated in the present study without addressing the problem of its stability. Earlier, Manankova and Pudovkin (1996) examined in detail the energetic parameters of the current sheet under uniform boundary conditions and showed that the free energy (or Helmholtz thermodynamical potential) of such a system is the same for both the uniform and filamentary state, i.e. it is impossible to distinguish between which of these two modi is energetically preferential. Performing a similar examination for the current sheet with the nonuniform boundary condition is a much more complicated problem, which is beyond the scope of the present study. In principle, in this case the two states of the current sheet may be non-equivalent energetically.

\section{Summary}

1. The solution of the Grad-Shafranov equation obtained in the present work is an exact analytical solution of the nonlinear problem which is determined by five independent parameters that characterize the state of the magnetospheric tail. The solution enables one to estimate self-consistently the effects of such factors as the current-carrying particle acceleration, temperature variation, the change in the integral cross-tail current, etc., in the configuration of the plasma sheet and magnetic field.

2. The possibility of obtaining an exact solution of the nonlinear equation (linearization of the Grad-Shafranov equation and transfer to the linear Schrödinger equation have not been used) allows one to apply this solution for the description of the most interesting transition region of the magnetosphere-region of a strong interaction of the geodipole and magnetotail current fields. This makes it possible to model self-consistently the structure, characteristic size of the transition region and its dynamics under quasi-stationary variation of the current sheet parameters.

3. It is known that for the description of the transition region of the magnetosphere one cannot use the models based on the approximation of a "slow variation" along the sheet (tail approximation) when $L_{x} \gg L_{z}$, where $L_{x}, L_{z}$ are characteristic scales of the change in all variables with the $x$ - and $z$-coordinates. Under the "taillike" approximation the solution has the form $A(z, \varepsilon x)$. The obtained exact solution of the 2-D problem for uniform boundary conditions along the sheet may be used to include (by means of the known scheme of the asymptotic expansion) a slow variation of the sheet parameters along the $\mathrm{x}$-axis and to construct a solution of the type: $A(x, z ; \varepsilon x, \varepsilon z)$.

4. The solution obtained can be used as an initial configuration in simulating dynamical processes in the magnetotail current sheet, as well as in testing the current sheet stability. Unlike the Harris layer which has essentially a different topology and can lead to singularities when being used as an initial approximation, the exact solution already contains the principal topological features of the internal and external current system interaction.

5. It should be noted that the boundary conditions used in the problem most completely reproduce the physics of the phenomena developing within the closer zone in the centre of the current sheet. This outlines the region in which the constructed simple model is plausible. It is in this region that the most important magnetospheric dynamical processes are known to develop. While considering the interaction of the two current systems, we excluded from the equations the current system of the inner magnetosphere, and took into account its contribution only via the boundary conditions.

And here arises a question on the limits of the applicability of the model problem under consideration. Of course, the model has a series of limitations. These restrictions naturally resulted from the fact that the boundary conditions, as has been said above, most adequately reproduce the physics of the discussed phenomenon only in the central part of the plasma sheet:

Let us consider some consequences.

(a) With the increase in the distance from the centre of the sheet $(z=0)$, the shape of the magnetic field lines for the system determined by the values of $|\gamma|>\left|\gamma_{c}\right|=3$ does not agree with the expected picture corresponding to the interaction of the two current systems: the geodipole and magnetotail current systems (Figs. 4d, $5 d)$.

(b) It is impossible to describe a current sheet disturbance corresponding to large values of $|\gamma|$ when $x_{R} \rightarrow x_{b}$ : the information on the interacting current systems given by the boundary condition is in this case insufficient for the consideration of the current sheet.

(c) Presented calculations (Figs. 4-5) make it possible to consider the configuration of the field (of the currents) for both $|\gamma|>\left|\gamma_{c}\right|$ and $|\gamma|<\left|\gamma_{c}\right|$. As was said above, the quasi-stationary evolution is modelled only as a series of stationary solutions for any fixed value of time. The process of the transition through the critical value of the parameter $\gamma$ in the frame of the model of the quasistationary evolution in this paper is not discussed in itself. This problem needs a special consideration.

Acknowledgements. This work was supported by the Russian Foundation for Basic Research, grant No 03-05-64865.

Topical Editor T. Pulkkinen thanks two referees for their help in evaluating this paper. 


\section{References}

Becker, U., Neukirch, T., and Schindler, K.: On the quasi-static development of thin current sheets in magnetotail-like magnetic fields, J. Geophys. Res., 106, 3811-3825, 2001.

Birn, J., Somer, R. R., and Schindler, K.: Open and closed magnetospheric tail configurations and their stability, Astrophys. Space Sci., 35, 389-402, 1975.

Braginsky, S. I.: On the behaviour of fully ionized plasma in the strong magnetic field, Sov. Phys. JETP (in Russian), 33, 645, 1957.

Fadeev, V. M., Kvarzhava, I. F., and Komarov, N. N.: The selffocusing of the local plasma currents, Nucl. Fusion (in Russian), 5, 202-209, 1965.

Harris, E. G.: On a plasma sheath regions of oppositely directed magnetic field, Nuovo Cimento, 23, 115-121, 1962.

Kan, J. R.: On the structure of the magnetotail current sheet, J. Geophys. Res., 78, 3773-3781, 1973.

Komarov, I. N.: Topology of stationary plasma configurations in crossed self-consistent fields: spatially periodical structures of plasma, Nucl. Fusion (in Russian), 3, 174-182, 1963.

Manankova, A. V., and Pudovkin, M. I.: Energy characteristics of a two-dimensional current-carrying plasma, Geomagnetism and Aeronomy (English Translation), 36, 426, 1996.

Manankova, A. V., and Pudovkin, M. I.: The description of a twodimensional current-carrying plasma sheet in the hydrodynamic approximation of a single-component plasma model, Geomagnetism and Aeronomy (English Translation), 39, N 1, 40, 1999.

Manankova, A. V., Pudovkin M. I., and Runov, A. V.: Stationary configurations of the two-dimensional current-carrying plasma sheet: exact solutions, Geomagnetism and Aeronomy (English
Translation), 40, N 4, 430, 2000a.

Manankova, A. V., Runov, A. V., Prosolin, V. I., and Pudovkin, M. I.: On two-dimensional steady-state non-uniform plasma sheet configurations. Proc. of the Fifth International Conference on Substorms (St.Petersburg, Russia, 16-20 May 2000), ESA SP443, 401-404, 2000b.

Manankova A. V. and Pudovkin, M. I.: Steady-state configurations of an inhomogeneous current-carrying 2D-plasma sheet: quasistationary evolution of the system, in: Proc. 4th Intern. Conf. "Problems of Geocosmos" (St.Petersburg, 3-8 June 2002), edited by Semenov, V. S., Lyatskaya, A. M., Kubyshkina, M. V., Biernat, H. K., 132-135, 2002.

Schindler, K.: A self-consistent theory of the tail of the magnetosphere, in: Earth's magnetospheric processes, 200-209, edited by McCormac, B. M., Reidel, D., Norwell. Mass., 1972.

Schindler, K. and Birn, J.: On the cause of thin current sheets in the near-Earth magnetotail and their possible significance for magnetospheric substorms, J. Geophys. Res., 98, 15 477-15 485, 1993.

Trubnikov, B. A.: Current filaments in plasmas, Plasma Physics (in Russian), 28, 346-359, 2002.

Tsyganenko, N. A.: A magnetospheric magnetic field model with a warped tail current sheet, Planet. Space Sci., 37, 5-20, 1989.

Vekua, I. N.: Notes on the solution properties of the equation $\Delta U=$ $-2 K e^{U}$, Siberian Math. Journ. (in Russian), 1, 331-342, 1960.

Vlasov, A. A.: A theory of new plasmoid, J. of Technical Physics (in Russian), 31, 785-796, 1961.

Walcker, G. W.: Some problems illustrating the forms of nebulae, Proc. R. Soc. London, Ser. A, 91, 410, 1915.

Wang, X. and Bhattacharjee, A.: An equilibrium model of the Earth's magnetotail: thin current sheet and a near-Earth neutral line, J. Geophys. Res., 104, 7045-7048, 1999. 